
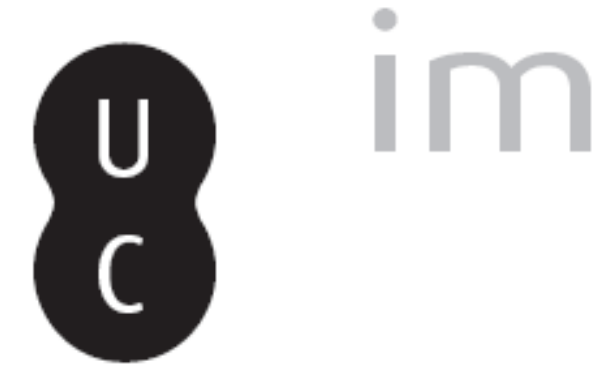

\title{
prartilim
}

\section{Eros e linguaggio nel Simposio}

\section{Autor(es): $\quad$ Palumbo, Lidia}

Publicado por: Annablume Clássica; Imprensa da Universidade de Coimbra

URL persistente:

URI:http://hdl.handle.net/10316.2/24308

DOI:

DOI:http://dx.doi.org/10.14195/1984-249X_9_8

Accessed : $\quad$ 26-Apr-2023 15:11:58

A navegação consulta e descarregamento dos títulos inseridos nas Bibliotecas Digitais UC Digitalis, UC Pombalina e UC Impactum, pressupõem a aceitação plena e sem reservas dos Termos e Condições de Uso destas Bibliotecas Digitais, disponíveis em https://digitalis.uc.pt/pt-pt/termos.

Conforme exposto nos referidos Termos e Condições de Uso, o descarregamento de títulos de acesso restrito requer uma licença válida de autorização devendo o utilizador aceder ao(s) documento(s) a partir de um endereço de IP da instituição detentora da supramencionada licença.

Ao utilizador é apenas permitido o descarregamento para uso pessoal, pelo que o emprego do(s) título(s) descarregado(s) para outro fim, designadamente comercial, carece de autorização do respetivo autor ou editor da obra.

Na medida em que todas as obras da UC Digitalis se encontram protegidas pelo Código do Direito de Autor e Direitos Conexos e demais legislação aplicável, toda a cópia, parcial ou total, deste documento, nos casos em que é legalmente admitida, deverá conter ou fazer-se acompanhar por este aviso.

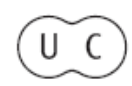




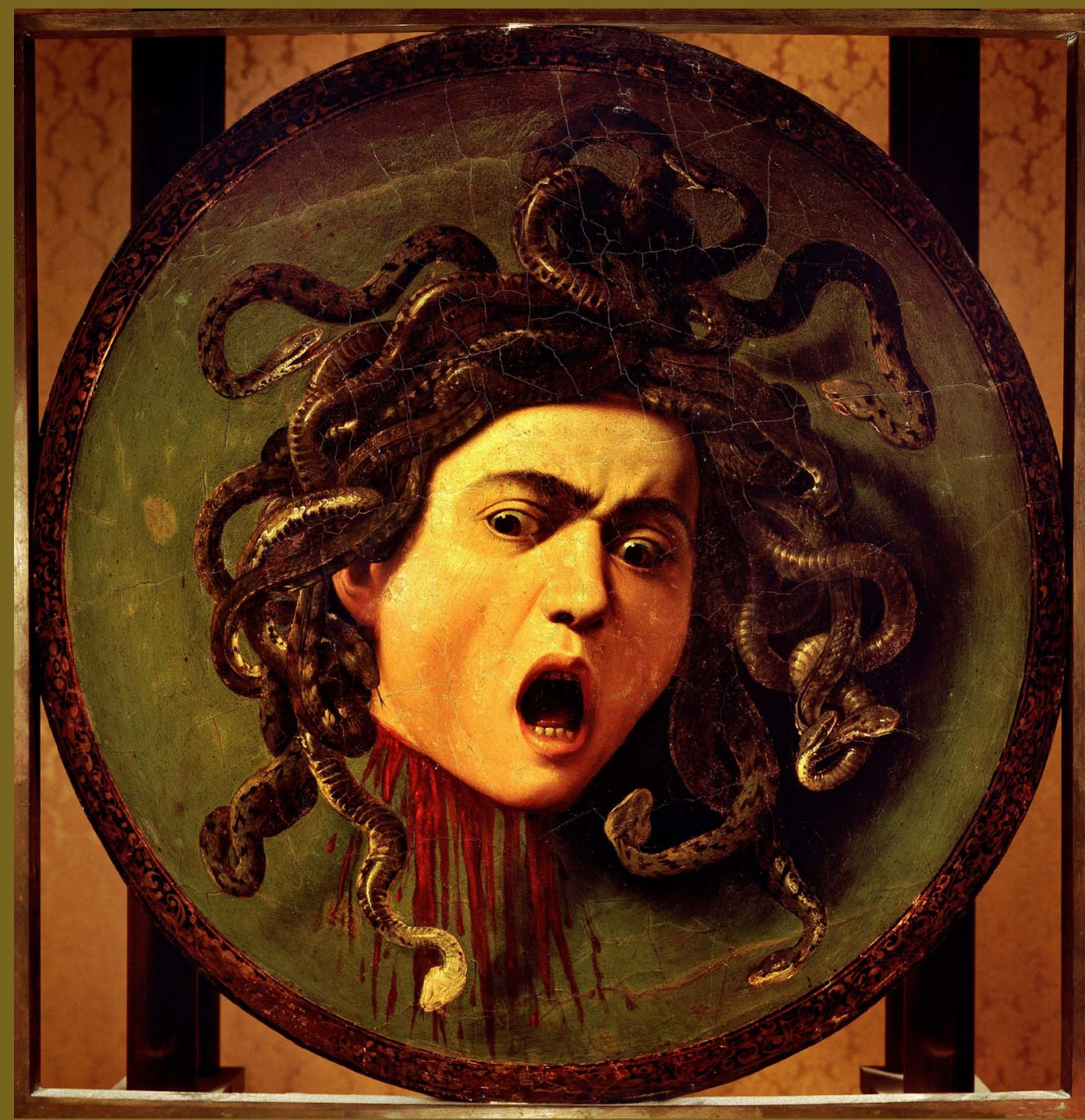

\section{R E V I S T A}
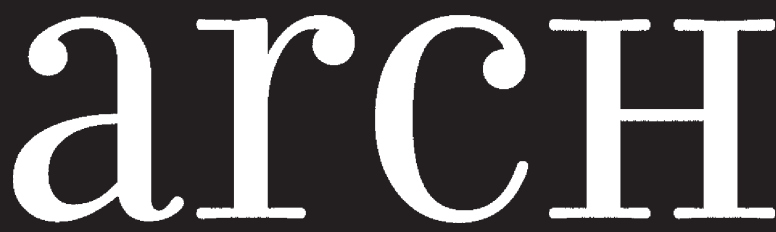
AS ORIGENS DO PENSAMENTO OCIDENTAL

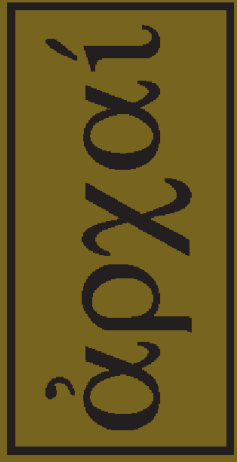

ARCHAI JOURNAL: ON THE ORIGINS OF WESTERN THOUGHT
arcHaI

arementam

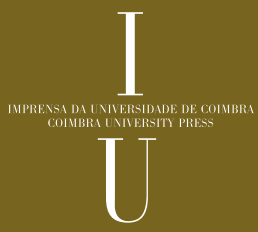

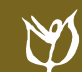

NNN 


\section{EROS E LINGUAGGIO NEL SIMPOSIO}

PALUMBO, L. (2012). "Eros e Linguaggio nel Simposio". Archai n. 9 , jul-dez 2012, pp. 85-92.

RIASSUNTO: In questa relazione suggerisco di considerare almeno la possibilità che nel Simposio Platone ci offre non una mera spiegazione della natura dell'amore, ma una spiegazione filosofica della natura del linguaggio (sull'amore). Nel Simposio Eros è una maschera di Socrate e Socrate una maschera del linguaggio. La storia di Diotima sulla nascita di Eros, figlio di Poros e Penia conferma questo punto: il linguaggio, come l'amore, non può possedere il suo oggetto come qualcosa di presente ma solo sempre in una fragile bilancia tra presenza e assenza, tra oblio e memoria, tra conoscenza e ignoranza. Il linguaggio, come l'amore, è il demone della comunicazione. In questa relazione presento qualche prova, tratta dal dialogo, atta a supportare tale interpretazione.

PAROLE CHIAVE: Simposio - Eros - Maschera - Socrate Linguaggio

ABSTRACT: In this paper I suggest to consider at least the possibility that in the Symposium Plato offers us not an account of the nature of love, but a philosophical account of the nature of language (or the language about love). In the Symposium Eros is a mask of Socrates and Socrates is a mask of language. Diotima's story regarding the parentage of Eros, child of both Poros and Penia, confirms this point: the language, as the love, cannot possess its object as something present but only always in a fragile balance between presence and absence, between oblivion and recollection, between knowledge and ignorance. Language, as the love, is the daimon of communication. In

\section{Lidia Palumbo*}

* Università di Napoli “Federico II", Napoli, Itália.

1. Per le quali, così come per alcune delle osservazioni che seguono, sono debitrice nei confronti dell'eccellente saggio di Moroncini (2010) dedicato alla lettura lacaniana del Simposio.
A Mimmo, carissimo e perduto per sempre perduto

$I_{n}$ questa relazione vorrei mostrare come sia importante, più che mai per il Simposio, fare attenzione alla complessità delle strategie testuali e non ridurre il dialogo ad uno schema concettuale (PUCHNER, 2010). Piuttosto che compiere una lettura che corra precipitosa verso l'individuazione dei significati, desidero, invece, in questa sede, indugiare sui significanti, riflettendo sui rimandi interni di un testo che rivela una straordinaria capacità di de-costruirsi: ognuno dei discorsi su Eros è criticato dagli altri discorsi, e il discorso di Alcibiade critica l'intero complesso dei discorsi precedenti, compreso quello di Socrate-Diotima. Quello che proverò allora a fare è spostare il fuoco dell'interpretazione, da un lato, allargando lo spettro ermeneutico e guardando non al singolo discorso pronunciato da ciascuno dei personaggi, ma ad alcuni collegamenti tra i diversi discorsi nel dialogo (e, all'occasione, anche ad altri dialoghi, che siano richiamati da una parola o da una curvatura del discorso) e, dall'altro, invece, restringendo il diametro ermeneutico per cogliere la singola espressione significante.

L'attenzione alle decostruzione del testo e la focalizzazione sulle parole ${ }^{1}$ mi fanno formulare 
this paper I offer some evidence in the dialogue for this interpretation.

PAROLE CHIAVE: Symposium - Eros - Mask - Socrates - Language

RESUMO: Neste texto, sugiro que se considere pelo menos como possivel que Platão esteja nos oferecendo no Simpósio não uma explicação filosófica acerca da natureza do amor, mas uma explicação filosófica acerca da natureza da linguagem (sobre o amor). No Simpósio, Eros é uma máscara de Sócrates e Sócrates uma máscara da linguagem. 0 relato de Diotima sobre o nascimento de Eros, filho de Poros e Penia, confirma este ponto: a linguagem, como o amor, não pode possuir o seu objeto como algo de presente, mas somente e sempre em um frágil balanceamento entre presença e ausência, entre esquecimento e memória, entre conhecimento e ignorância. A linguagem, como o amor, é o daimon da comunicação. Neste texto, apresento algumas provas, extraídas do diálogo, aptas a sustentar tal interpretação.

Parole chiave: Simpósio - Eros - Máscara - Sócrates - Linguagem

un'ipotesi di lettura del Simposio che, con tutte le cautele che è doveroso assumere in casi come quello di questa interpretazione ${ }^{2}$, prova ad individuare per così dire una trama nascosta sotto una trama apparente, un messaggio nascosto sotto un messaggio apparente, un argomento nascosto sotto un argomento apparente ${ }^{3}$, e a leggere il dialogo come la tensione dei dialoganti a cercare non già, o non solo, o non primieramente, lo statuto di Eros, ma piuttosto lo statuto del linguaggio (su Eros).

Come Socrate ${ }^{4}$, la cui storia, nel Simposio (CORRIGAN, 2004) come nel Fedone, ci viene significativamente raccontata dai suoi amanti ${ }^{5}$, appare, nell'immagine che viene presentata da Alcibiade in Symp. 215A-B, dotato di una sembianza esteriore che contrasta con l'essenza interiore, di un fuori in contrasto con il dentro; allo stesso modo il racconto su Socrate fatto da Apollodoro potrebbe presentare un significato nascosto diverso da quello apparente: dello statuto delle parole, e della loro tensione ad esprimere la verità, potrebbe in realtà trattare il Simposio ed Eros potrebbe rappresentare solo l'argomento apparente, l'occasione discorsiva che consente al vero tema del dialogo, in qualche modo nascostamente, di articolarsi ${ }^{6}$..

Questa proposta di lettura del testo, che vado nelle prossime pagine ad argomentare, si riferisce ad una modalità antica di interpretazione dei racconti - e il Simposio nella sua interezza può essere descritto come un racconto - che risale almeno ad Antistene ${ }^{7}$, che sosteneva che il racconto dovesse essere compreso considerando che in esso vi è un senso apparente (doxa) e uno vero (aletheia).

La prima questione esplicitamente teorizzata da Socrate quando egli arriva a casa di Agatone, prima ancora cioè di sdraiarsi e cenare insieme agli altri, prima ancora di fare le libagioni, i canti e gli altri atti di rito, è la questione del sapere. Socrate e Agatone, portatori ciascuno di una forma di linguaggio, rappresentano due possibilità del sapere: Agatone è la possibilità del sapere tragico, descritta con una serie di termini indicanti luce e scintillìo; Socrate, invece, è la possibilità di un sapere controverso (amphisbetesismos, 175E) e dubbio come un sogno. A me sembra che il primo possa rappresentare il linguaggio preso nella sua esteriorità poetica e retorica, e che il secondo, invece, possa rappresentare il linguaggio nella sua dimensione profonda: quella nascosta, la cui struttura, ambigua e difficilmente afferrabile, è affidata ai significanti. È con ironia che Socrate afferma:

$\grave{E}$ un vero onore per me stare sdraiato accanto a te: così, credo, mi colmerai di copiosa e splendida sapienza. In realtà la mia, seppure esiste, è modesta o almeno inconsistente come un sogno (hosper onar), quanto invece è luminosa (lampra) e piena di promesse la tua che, nonostante la tua giovane età, è già sfolgorata ed è apparsa in piena luce appena l'altro ieri alla presenza di più di trentamila Elleni (175E).

Il sapere di Agatone, come il suo linguaggio, è esteriore ed appariscente, è fruito da grandi moltitudini, laddove quello di Socrate, compreso da pochissimi, è onirico: da decifrare ${ }^{8}$.

Dopo che questa questione preliminare, tesa forse ad invitare il lettore alla decifrazione del significato del testo, è stata posta, e il banchetto (syndeipnon) ha ceduto il passo al simposio (sym-
2. Conformemente a quanto raccomanda Szlezák (1991).

3. Per l'idea che Platone ci nasconde quel che pensa nel momento stesso in cui ce lo rivela e che nella presentazione $\mathrm{d}$ quello che si può chiamare il suo pensiero abbia deliberatamente preservato il posto dell'enigma si veda quanto scrive Lacan (2008), p. 69 , p. 184

4. Che - come si vedrà - è Eros ed è linguaggio, ed è eros che ha per oggetto il linguaggio, ed è linguaggio che ha per oggetto eros .

5. Quando, nel Fedone, Echecrate chiede a Fedone di narrare la morte di Socrate, egli acconsente subito, e con gioia, perché, dice, "ricordarmi di Socrate, parlandone io stesso o sentendone parlare da altri, è sempre per me la pi dolce di tutte le cose" (Phaed. 58D5-6). È indubbio che Platone intende presentare Fedone come innamorato di Socrate: soltanto quando si è innamorat di qualcuno, infatti, accade che ricordarci di lui sia sempre la più dolce di tutte le cose (panton hediston). Ed è significativo che Platone, nel costruire il racconto della morte del maestro, scelga di affidarlo ad un narratore tutt'altro che neutrale; ciò consentirà al racconto stesso, infatti, di assumere non la forma della storia verosimile, resoconto di accadimenti, ma quella della narrazione simbolica. Appartenendo all'universo del simbolo, l'evento della morte di Socrate si configura come qualcosa il cui significato trascende se stesso per diventare qualcosa d'altro, qualcosa di tutti, di universale e al contempo di intimamente individuale; sull'argomento Palumbo (2003), pp. 291-300). Affidare ad un amante di Socrate il compito della narrazione ha, nel Simposio, un effetto simile a quello che si genera nel Fedone: la storia di Socrate diventa occasione di riflessione, spazio verbale tutto da vivere per condurre ciascuno verso se stesso.

7. Non a caso i due dialogh dedicati all'eros, il Fedro e il Simposio, sono entrambi dedicati, nel punto chiave dell'argomentazione, a mostrare come il discorso debba incontrare il suo oggetto, e cioè la verità: come un logos debba dire ciò che è. Phaedr. 277B, Symp. 199B. 
7. Antist. ap. Dion. Chrys. Or. LII 4, come ricorda Rudhardt (1999), p. 27.

8. La distinzione tra esteriorit svalutata e interiorità preziosa si ritrova anche nel passo sulle statuette dei sileni, che potrebbe star lì, nel discorso di Alcibiade $(215 \mathrm{~A})$, a rappresentare un metafora di Socrate e, attraverso Socrate, dell'intero dialogo.

9. Soph. Ant. 781-800, Eurip. Hipp. 525-35, Med. 835sgg. Per encomi in prosa si può pensare a produzioni proprio della cerchia dei socratici. Sull'argomento Fasce (1977).

10. Una interpretazione in questa prospettiva quella di Casertano

(1997).

11. Una lode del potere de linguaggio, non a caso anch'essa collegata al tema dell'amore, è l'Encomio di Elena di Gorgia. Platone intende sistematicamente riscrivere, rovesciandone la

forma, le composizioni poetiche e sofistiche, e se il mito di è leggibile come la versione platonica della Nekija omerica (Sull'intenzione platonica di sostituire i testi poetici tradizional con composizioni nuove: Cerr 2000), allo stesso modo i Simposio può essere letto come la riscrittura dell' Elena gorgiana: la parola filosofica sul rapporto tra eros e linguaggio contro parola sofistica. A far sospettare di questa vis polemica antisofistica specificamente indirizzata contro il Gorgia dell' Encomio sta non soltanto la esplicita citazion di Gorgia alla fine del discorso di Agatone (198C), ma anche tutta la disquisizione su come si fa un encomio che segue suddetta citazione fino a $199 \mathrm{C}$. Sui personaggi del Simposio che figurano muti nel Protagora e sul loro legame con i sofisti cfr. Deschoux (1980), p.198.

12. Eros e linguaggio intessono entrambi una relazione con la carenza, con l'aporia, ed entrambi possiedono nella loro aporia la risorsa che li condurrà all'euporia cui aspirano. Sulla risorsa già presente nella aporia di Penia cfr. Sheffield 2006, p. 60.

13. Zefiro ed Iri, secondo Alceo (fr. 13 Bergk); Ares e Afrodite, secondo Simonide (fr. 43 Bergk), Urano e Gea, secondo Saffo (fr. 133 Bergk), Zeus secondo Euripide (Hipp. 534); Nucci (2009), p. 33, n.59. posion), Erissimaco, facendosi portavoce di una proposta di Fedro (177A), chiede che ci si dedichi ad un encomio di Eros. Da quel che dice Erissimaco è già però possibile sospettare che egli stia parlando di Eros, intendendo però riferirsi ad altro. Egli dice infatti che ci sono inni e peani composti per gli dei e non ce n'è per Eros: nessun poeta - dice Erissimaco - ne ha composti. Ma, come tutti sanno, questo non è vero, perché encomi in prosa e in versi su Eros ne esistevano, e come ${ }^{9}$. I sofisti - dice Erissimaco - non ne hanno composti, eppure ne esistono tanti su tanti altri soggetti, per esempio sul sale.

Io credo che questo riferirsi all'assenza di una cosa di cui si conosce invece la presenza sia una spia testuale che allude al fatto che è un'altra cosa ciò di cui si lamenta l'assenza e di cui ci si appresta a parlare. Si lodano le cose le più diverse, si riflette cioè sull'utilità di esse (177B6), addirittura si loda il sale, e non si loda (non si riflette sull'utilità di) ciò che ogni lode rende possibile.

Come proverò ad ipotizzare, ciò di cui si denuncia l'assenza potrebbe essere una vera riflessione filosofica sul linguaggio ${ }^{10}$, e il fatto che si citino $i$ sofisti potrebbe essere un riferimento alle loro riflessioni sul logos, riflessioni che Platone in questa sede ignorerebbe per marcare un rifiuto, intendendo riscriverle completamente ${ }^{11}$.

In 177D Socrate afferma di non conoscere nulla, se non ta erotika, le questioni erotiche. Anche questa potrebbe essere un'affermazione leggibile nella direzione indicata, infatti il non sapere nulla è un mancare che prelude al cercare, traccia che conduce a disegnare tanto la figura dell'eros (e non solo nel racconto su Poros e Penia) quanto quella del linguaggio, inteso come tensione euristica all'espressione di una significazione. Comincerebbe così ad essere posta quella sovrapposizione, o sovrapponibilità, delle figure del linguaggio e dell'eros ${ }^{12}$ che caratterizzerà l'intero discorso di Socrate.

Apollodoro dice che Aristodemo, la persona da cui egli ha appreso il racconto del simposio dedicato agli erotikoi logoi, alcuni discorsi li ricordava meglio e altri peggio, e lui stesso poi, che ora sta raccontando, alcune cose le ha tenute a mente e altre no $(178 \mathrm{~A})$. Si tratta di una riflessione sulla pratica di produzione del dialogo, del discorso, e del racconto di discorsi (MORONCINI, 2010); riflessione che, lungi da essere accessoria, potrebbe star lì, nel testo di Platone, per alludere, per così dire, all'argomento cruciale tematizzato dalla discussione.

Il primo discorso - come è noto - è quello di Fedro. Egli è detto padre del discorso (pater tou logou, 177D), perché ha proposto l'argomento, ma anche perché dell'argomento affronta, come un padre, la genesis (178A10). Eros - egli dice - è la più antica delle divinità e la sua antichità è segno del suo onore. Prova di questa antichità onoranda - dice Fedro - è il fatto che non se ne conoscono i genitori, "né vengono nominati da nessuno, né prosatore né poeta", 178B3-4. Ancora una volta, allora, è possibile sospettare che non è di Eros che si sta parlando; il mito testimonia, infatti, in vario modo, dei genitori di $\operatorname{Eros}^{13}$. In maniera nascosta qui Platone potrebbe star forse allora dicendo non che non conosciamo le origini di Eros, ma che non conosciamo le origini di una cosa altra che non viene nominata; che di essa sappiamo solo che è antichissima, ed è per noi causa di grandissimi beni ${ }^{14}$.

D’altra parte i primi discorsi nel Simposio sono irriflessi, portatori cioè di concezioni che per essere coerentemente comprese, filosoficamente comprese, devono essere criticate, ed infatti puntualmente ogni discorso critica il precedente, fino al discorso di Socrate, che è invece trasparente a se stesso e che, attraverso la critica ad Agatone, critica tutti i discorsi precedenti e rappresenta nel testo una vera e propria frattura: l'instaurazione di una nuova procedura discorsiva.

Quando prende la parola Socrate, tutto cambia. Hanno già parlato Fedro, con il suo carico di citazioni poetiche, Pausania, con i suoi riferimenti alla paideia, ha parlato Erissimaco il medico, poi Aristofane il commediografo e infine Agatone il tragediografo. Finora uno parlava e gli altri ascoltavano; ora che parla Socrate, invece, gli interlocutori cessano di essere passivi ascoltatori e vengono coinvolti in un dialogo. Socrate comincia, infatti, ad interrogare Agatone e l'interrogazione mostra tutta la sua natura erotica, essendo arte espressiva, tentativo inesausto di portare alla luce un sapere vero che, secondo il modello del Menone, si nasconde nell'inconsapevolezza e chiede solo che un maestro, 
un amante ${ }^{15}$, sia capace di farlo venir fuori. Ma non solo. Questo sapere vero mostra immediatamente la sua natura linguistica: è un sapere fatto di segni e di relazioni necessarie tra segni.

E allora, su Eros, visto che per il resto hai spiegato con bellezza e magnificenza quale è, dimmi anche questo: Eros è di natura tale da essere eros di qualcosa o di nulla? (tinos eros e oudenos) Non chiedo se lo sia di una madre o di un padre. Sarebbe ridicola infatti la domanda se Eros sia di una madre o di un padre. Invece, come se proprio di questo - del padre - ti chiedessi: il padre è padre di qualcuno o no? Mi risponderesti certamente, se volessi rispondere bene, che il padre è padre di un figlio o di una figlia. 0 no?

Certo, disse Agatone.

E anche per la madre è lo stesso?

Fu d'accordo anche su questo.

E allora - disse Socrate - rispondi ancora un po', per capire meglio ciò che voglio. Se infatti domandassi: un fratello, in quanto fratello, è fratello di qualcuno o no? Confermò che era così: è fratello di un fratello o di una sorella.

E allora cerca di dirlo anche per Eros. Eros è eros di nulla o di qualcosa?

Di qualcosa, certo. (199D-E).

È impossibile sopravvalutare l'importanza di questo passo: con un unico gesto, ed immediato, Socrate cancella tutto il variopinto mondo del mito, della tradizione poetica, politica, medica, che aveva caratterizzato i precedenti discorsi, tutto il loro riferirsi all'esperienza personale degli affetti pederastici, della loro portata paideutica, etica, retorica, cancella le curvature comiche e tragiche dei precedenti discorsi e si riferisce alla purezza del significante in questione: eros è eros di qualcosa o di nulla? La domanda socratica su eros tende a focalizzare una struttura di rinvio, non ancora il rinvio di un significante ad un significato, ma il rinvio del significante ad un altro significante (MORONCINI, 2010) $)^{16}$ : "eros" rinvia necessariamente a "qualcosa", come "padre" rinvia a "figlio". Padre, però, rinvia ad un significante determinato, rinvia a "figlio"; "eros", invece, rinvia a qualcosa. È difficile immaginare un termine più linguistico di "qualcosa". Si tratta di un termine che compare in un discorso solo quando l'argomento del discorso è squisitamente linguistico, quando il discorso è una riflessione sulla natura della lingua, quando ciò di cui si sta parlando è la possibilità stessa di parlare e di costruire discorsi. Non c'è padre senza figlio, argomenta Socrate, come non c'è amore che non sia amore di qualcosa. Un amore di nulla, come un padre di nulla, non sarebbero né un amore né un padre. Il discorso di Socrate all'inizio sembra avere il tenore del trattato grammaticale, del gioco linguistico, della struttura enigmatica: è quel modo di tendere la mente alla comprensione del potere delle parole che toglie tragicità ai discorsi e li colloca in un orizzonte senza tempo e senza vecchiaia che aiuta ad entrare nell'orizzonte della filosofia.

In questo orizzonte le parole sono tutte legate ad una struttura di rinvio che è la rete del linguaggio: padre rinvia a figlio, significa solo se rinvia al significante figlio e all'intero tesoro dei significanti che quest'ultimo implica. Amore rinvia a qualcosa: significa solo se rinvia ad un qualsiasi altro significante, vale a dire all'intera costellazione delle parole che in una data lingua possono collegarsi alla tensione di eros a significare. In questo senso, allora, eros nel discorso di Socrate non è solo un significante, ma un significante che significa ogni altro significante (MORONCINI, 2010) ${ }^{17}$, che rappresenta la struttura stessa della lingua, con i suoi rinvii reciproci, con la sua tensione ad esprimere, con la sua dipendenza dal "qualcosa": è l'occasione per riflettere sul linguaggio e sulla sua natura erotica 0 , ciò che è lo stesso, su eros e sulla sua natura linguistica.

Pronunciata da Socrate, questa identificazione tra eros e linguaggio non ci sorprenderebbe. Nel Fedro, infatti, collegando l'amore alle diaireseis, alle operazioni di scomposizione compiute dal logos impegnato a pensare le cose per comprenderle, il filosofo ha dichiarato di esserne sempre stato innamorato.

Io, Fedro, sono amante di questi procedimenti, delle divisioni e delle unificazioni, al fine di essere in grado di parlare e di pensare; e se ritengo che qualcun altro sia per sua natura capace di guardare all'uno e ai molti, lo
14. Di questa cosa altra i discorsi del Simposio potrebbero star tracciando una serie di elementi tesi a disegnare una figura che apparirà a chi sappia leggere $i$ messaggi di ciascuno dei discorsi.

15. Sulla sovrapponibilità della figura del maestro e di quella dell'amante cfr. i testi sulla pederastia. Una bibliografia in Calame 1992.

16. (p.119) Nell'ipotesi di lettura che sto provando a proporre l'argomento del Simposio è una riflessione sulla natura del linguaggio. In tal senso esso è un sistema di segni, una rete di significanti che rinviano innanzitutto l'uno all'altro e poi, in secondo luogo, ad un mondo di significati che stanno, rispetto ai significanti, come gli amati rispetto agli amanti. Ciò consente di annotare come sia l'amore sia il linguaggio siano strutture di rinvio.

17. (p. 122). Come appare dagli studi di Rudhardt (1999), il significato più antico dell'eros è quello che riconosce in esso la funzione espressiva, quella capace di portare alla luce - come appare nella Teogonia di Esiodo - ciò che oscuramente si nasconde nel grembo delle entità primordiali. 
seguo "tenendo dietro alle sue orme come a quelle di un dio (Phaedr. 266B).

Se in questo passo del Fedro Socrate dichiara di amare le operazioni linguistiche, qui nel Simposio dichiara (177D) di possedere un sapere solo sulle cose d'amore; continuamente accenna, cioè, in modo oscuramente allusivo, alla possibilità di apparentare eros e logos.

Ottenuto da Agatone, suo amato, l'assenso sulla relazionalità intrinseca del termine eros, Socrate lo invita a custodire dentro di sé questo primo sapere sull'amore. E continua ad interrogare.

Questo allora custodiscilo dentro di te e ricordatene. Dimmi invece: Eros, quello di cui è Eros, lo desidera o no? Certo.

8. Ciascuno - dice Aristofan - cerca sempre il simbolo di se stesso (191D6). Ogni ente cerca, in uno specifico altro ente, insieme con il quale forma un intero, la maniera di esprimere se stesso, e tale tensione, tale tou holou epithymia (192E) è pe lui qualcosa come la strada della felicità.

19. Interamente linguistica anche tale specificazione, essa avviene, infatti, tutta sul piano del linguaggio, nello spazio che esiste tra il significante e ciò cui esso sempre necessariamente rimanda.

20. Il riferimento al vissuto non è negato nel senso che venga ignorato. Esso, secondo una modalità del pensiero che si può dire anche peculiarmente platonica, tesa a conseguire le due finalità del pensiero dialettico, viene, con un unico movimento, tolto e conservato: tolto, affinché la comprensione del fenomeno possa essere allargata a dati altri dal particolare in questione, conservato, affinch non vengano perdute "vita, anima ed intelligenza" (Soph. 248E) che l'essere non può mancare $\mathrm{d} i$ avere se vuole essere compreso. Sull'argomento Pimenta (2006),

21. Come sottolinea Moroncin (2010), p. 124, Agatone giunge alla incontrovertibile verità in 201C come a suo tempo vi era giunto lo schiavo di Menone, solo seguendo la catena de significanti.
Ma possedendo quello che desidera e ama, poi lo desidera e ama, oppure non possedendolo?

Non possedendolo, a giudicare in base al verosimile

Considera - disse Socrate - se invece che verosimile non sia necessario questo: chi desidera, desidera ciò di cui è privo, oppure non desidera, se non è privo? A me infatti, Agatone sembra meravigliosamente che sia necessario. A te? (200A-B).

Socrate non parla di sé, né parla degli altri. L'argomento del suo discorso è "chi desidera" (ho epithymon) e poi anche la relazione che "chi desidera" intesse con "ciò che desidera". Esiste qualcosa di fisso ed immutabile, qualcosa di necessario, che accomuna, prescindendo da tutte le possibili variazioni modulari, il desiderante e il desiderato. Questo qualcosa è la struttura della mancanza. Tale struttura è ciò che lega l'amante all'amato, è ciò che dà al primo un significato che lo lega al secondo dandogli significato: entrambi, il desiderante e il desiderato, hanno significato solo nella relazione reciproca ed insieme formano un intero, la verità sull'amore: come i due symbola di cui parla Aristofane; ed è difficile, ancora una volta, pensare ad un termine più linguistico di symboln ${ }^{18}$.

Isolata la struttura della mancanza - il non avere (ouk echein) - come la verità necessaria dell'eros epithymetico, Socrate si interroga sulla eventualità che possa darsi una forma di eros anche nel caso in cui manchi al desiderante proprio la mancanza (200B7), nel caso, cioè, in cui il desiderante possiede ciò che desidera. Ma questo caso viene considerato adunaton (B6).

La contemplazione di casi possibili, la esclusione di alcuni di essi sotto il segno dell'adunaton, l'individuazione di predicazioni attribuibili secondo la modalità della necessità, della possibilità o della verisimiglianza sono modulazioni argomentative che collocano il discorso di Socrate in un registro molto diverso da quello dei discorsi precedenti, un registro - è una connotazione che va specificandosi sempre di più - prettamente linguistico e semiotico, teso com'è alla individuazione di semeia, di segni, che mostrino non tanto che cosa sia l'eros, ma come sia impossibile parlarne senza parlare innanzitutto delle parole, e della loro tensione a significare, tensione che esse esplicano riferendosi l'una all'altra in un tessuto di segni che consentirebbe di ricostruire, se lo si seguisse interamente, l'interezza della lingua.

Sarà necessario introdurre la variabile temporale per comprendere che si possono desiderare anche cose che si posseggono; in questo caso, infatti, si desidererà di possederle in futuro, e il riferimento al futuro agisce come un dispositivo di conferma delle affermazioni già fatte ${ }^{19}$ : è sempre e solo il mancare che genera l'epithymia, il mancare che, proiettato nel futuro, fa sì che ciò che ora possediamo ci possa già mancare, già riempirci di nostalgia, nostalgia proiettiva e retrospettiva, amore di ciò che può scomparire, perché tutto un giorno scomparirà, o scomparirà per noi: scompariremo infatti noi stessi e qui il discorso su eros incontrerebbe il discorso su thanatos se Socrate non lo impedisse, con una virata immediata, che impedisce ogni riferimento al vissuto ${ }^{20} 0$ a contenuti empirici, e focalizza l'attenzione del discorso su se stesso, sulla sua natura di luogo delle parole, dei significanti puri $^{21}$, legati in modo puro ai loro oggetti. Spogliati di ogni tragicità e di ogni dolore, di ogni forma e di ogni colore, secondo i dettami della dialettica (210E-212A).

È il momento del dialogo in cui è necessario provare a riassumere quanto già detto e, poiché è Socrate a fare questa prova, essa assume la forma della domanda: 
E costui, allora, e chiunque altro è desiderante (ho epithymon), è desiderante di ciò che non ha a disposizione e che non è presente (tou me parontos), di ciò che non ha (ho me echei) e ciò che non è egli stesso (ho me estin autos) e di cui è privo. Di questo genere (toiauta) sono gli oggetti del desiderio e di eros? (200E).

L'insieme delle caratteristiche dell'oggetto del desiderante presenta due tratti meritevoli di attenzione: il primo è quello che si raccoglie attorno all'affermazione che ciò che il desiderante è in se stesso (ho estin autos) non può essere oggetto del suo desiderio. Qual è il senso di questa affermazione? Essa vuole sottolineare che non può esistere un desiderio rivolto a se stessi? 0 , più precisamente, che non può esistere un desiderio rivolto a se stessi se il desiderio nasce da una mancanza, o da una mancanza possibile, temuta, futura. Ciò che un soggetto è, ciò che appartiene alla sua essenza, insegna il Fedone (103D-104B.), è qualcosa che a quel soggetto, finché esso resta tale, non può venire a mancare, pena la sua stessa scomparsa: la propria identità è qualcosa che non si può perdere e dunque - questa è la conseguenza dell'argomentazione - non la si può amare, perché si ama solo ciò che si desidera, e si desidera solo ciò che si può perdere, dunque ciò che ci accompagna in modo precario, provvisorio, ciò che possono sottrarci, che può venire a mancarci ${ }^{22}$.

Non amiamo ciò che ci caratterizza in modo essenziale, che appartiene a noi da sempre: non si può in nessun caso amare se stessi. L'eros è figura declinabile solo nel segno dell'heteron, mai del tauton, o, più precisamente, è figura declinabile solo nel segno del pros allo, mai del kath'hautò (Soph. 255C.).

Il secondo tratto interessante della schedatura delle caratteristiche del desiderante riguarda il più ambiguo dei dimostrativi: il termine toiouton. A questo termine è qui demandato il compito di individuare ciò che accomuna i diversi casi elencati, e li rende oggetti di desiderio: ciò che il desiderante non ha a disposizione, ciò che a lui non è presente, ciò che egli non ha, e ciò che egli non è e di cui è privo. Ora, come sottolinea Ledesma (2008), l'aggettivo dimostrativo toioutos-toiaute-toiouton è impiegato per alludere alla somiglianza che esiste tra cose che si lasciano ricondurre ad uno stesso "insieme"- in questo caso l'insieme delle cose desiderabili - ma non per questo esso designa "l'insieme", esso designa, al contrario, ciascuno degli elementi dell'insieme dotato della caratteristica per cui è elemento dell'insieme. L'aggettivo lo designa attraverso una comparazione che allude ad una somiglianza che accomuna. Ma che cosa sia ciò che accomuna, con il termine toiouton, è sottinteso non definito. Il termine funziona come una scorciatoia allusiva che tronca un elenco sfumandolo: le cose desiderabili sono quelle che non si hanno a disposizione, che non sono presenti, che non abbiamo, di cui siamo privi, e toiauta "le cose di questo genere ${ }^{\prime 23}$.

Ad un certo punto, come di consueto, Socrate riepiloga ciò che ha detto: Proton, Eros è amore di qualcosa, epeita è amore di ciò di cui avverte la mancanza (200E). Impossibile non notare che le parole più importanti sono proton ed epeita, le parole cioè che scandiscono il tempo logico dell'argomentazione, la scala di importanza della significazione, le parole che danno la misura di quanto stiamo progredendo nel campo della comprensione non già dell'eros, ma del nostro modo di rappresentarcelo. Prendendo a prestito il vocabolario dell'approccio ermeneutico alle passioni, è possibile affermare che l'eros non si configura in questa parte del testo del Simposio come un oggetto, ma come una parola, come un costrutto teorico applicato dagli uomini ad aree del loro vissuto per dare ad esse una voce ${ }^{24}$.

Ma è tempo di passare al discorso di Diotima (201E) e alla potente affermazione relativa ad Eros inteso come metaxy, come intermedio, tra sapienza e ignoranza. Socrate racconta di aver appreso dalla sacerdotessa elementi di un sapere squisitamente logico-linguistico, relativo innanzitutto alla semantica della negazione, che non va intesa come contrarietà ma come differenza ${ }^{25}$; relativo in secondo luogo alla corretta comprensione del corretto opinare (to ortha doxazein), che, essendo alogon, non può essere episteme, ma accadendogli di incontrare l'essere non può essere amathia. E relativo, in terzo luogo, alla maniera in cui vanno articolate le affermazioni sul divino. Le tre cognizioni portano Diotima a definire Eros un daimon, ed è questa, forse, la parte del discorso della sacerdotessa che più autorizza a leggere
22. Il desiderio indica una mancanza. Sempre e comunque. Anche quando, infatti, esso si riferisce a ciò che si possiede già, in quanto desiderio esso è costruito sull'immagine di mancanza futura che il desiderante si prospetta. Anche l'affermazione che l'amante è privo di bellezza, come sottolinea Price (1989), p. 19, è da intendersi come a dire che lo è in quanto amante. Il fatto di considerare il desiderio - o qualsiasi altra cosa - in quanto tale, è la marca del linguaggio filosofico che guarda all'essenza. Si tratta di un punto fondamentale. Guardare per esempio ad un medico non in quanto individuo empirico che cura questo o quello degli ammalati (compiendo errori umani), ma in quanto medico, in quanto curatore (therapeutes), che quindi non sbaglia, come appare in Resp. 341C, significa riflettere sul linguaggio inteso come sistema di significanti puri, ed è il compito squisitamente dialettico che contraddistingue per esempio le diaireseis che compaiono nel Politico e nel Sofista. Non si tratta di astrazioni di riflessioni sulla modalità della predicazione separata dal reale concreto e dal vissuto empirico orizzonti senza il riferimento ai quali nessuna riflessione sui significanti ha senso, ma si tratta dell'instaurazione di quel regime dialettico di continuità e di rottura tra essere e linguaggio che rappresenta la cifra più caratteristica che il platonismo ha donato alla storia della filosofia.

23. Qui nel Simposio il termine serve a sfumare la nozione di desiderabile, come nella Repubblica serviva a sfumare la nozione di immagine: "Per immagini intendo in primo luogo le ombre, poi i riflessi nell'acqua e in tutti i corpi compatti, lisci e lucidi, e ogni fenomeno del genere (pan to toiouton), se comprendi ..." (Resp.509D10-510A3).

24. Molti pensano - scrive Moravia (1995), p. 4 - che la passione sia una realtà a suo modo oggettiva, con la sola peculiarità (non di rado vivamente deprecata) di abitare entro il complesso universo del soggetto-uomo. Non è vero. La passione non esiste. Non esiste, voglio dire, nel modo in cui esiste una 'cosa' - sia pure una 'cosa' interna a noi. Nessuno ha mai colto esaustivamente la passione esplorando l'essere umano con strumenti tecnici 
quanto si voglia sofisticati. $E$ in verità ciò sorprende assai poco. Presto, assai presto, ci accorgiamo infatti che la 'passione' è, in prima approssimazione, una parola, un concetto. È, più esattamente, un costrutto teorico, connesso a matrici e fini plurimi, che l'uomo applica a una determinata are di vissuto per evidenziarne certi tratti e dar loro un significato, un voce. Secondo questa prospettiva, la passione (beninteso non solo essa) è un grande apparato significante.

25. Esattamente come in Soph.257B-C: ciò che non è sapiente non è ignorante (Symp. 202A).

26. Come sottolinea Moroncini (2010), p. 136, Poros privo di Penia era verità senza sapere, senza coscienza (in sogno) senza mancanza e dunque senza desiderio. L'aporia fa dono a Poros della sua mancanza ed ecco che può nascere la filosofia. Poros è un dio, Eros un demone: è la scala che scende quella istituit da Penia.

27. Eros non è né bello né delicato, questi tratti infatti spiega Diotima - sono dell'amato non dell'amante, e l'amato si sa - in quanto amato - è perfetto, invece perfetto non è l'amante, proprio in quanto amante.

28. Sarebbe interessant approfondire la questione della natura linguistica della scala amoris, che sembra ricalcare il procedimento per il quale, riconoscendo l'identico ne

diverso, e riconducendolo ad un'idea unica, è possibile attribuire ai molti oggetti un identica denominazione (cfr. Resp.X 596A). Anche qui, infatti, si tratta di ritrovare l'identica bellezza nei molti corpi belli, tale molteplicità da ricondurre all'unità va allargandosi dai molti corpi belli alle anime belle, a comportamenti, alle leggi, alle conoscenze, secondo un percorso che non è - come potrebbe sembrare dalla raccomandazione a disprezzare l'attaccamento ad un unico corpo in $210 \mathrm{~B}$ - di abbandono dell'unità verso la molteplicità, ma, al contrario, $\mathrm{d}$ ricerca della unità vera, unità della forma, unità della molteplicità, che è tanto più vera, più in alto nella scala, quanti più elementi empirici diversi, grazie all'unica denominazione, sono stati
Eros come la grande metafora linguistica dei dialoghi platonici dedicati all'amore: Eros infatti hermeneuei kai diaportemeuei, è "interprete e messaggero", e si colloca tra gli uomini e gli dèi. La sua posizione di intermedio e di intermediario rende il tutto un intero, qualcosa le cui parti sono collegate le une alle altre. Sembra davvero una descrizione ravvicinata della natura del logos: ogni comunicazione col divino avviene attraverso un daimon che dunque è esso stesso per natura un linguaggio, un linguaggio atto ad avvicinare, a legare (syndedesasthai 202E7), a far comunicare quelle parti che, avvicinate, formano un tutto; e tale loro avvicinarsi - come nel caso di ogni ermeneutica - è riempimento di una distanza (sympleroi 202E6), quella che impedisce la comprensione: ed ecco generarsi la mantica, l'arte dei sacerdoti, dei sacrifici, dei misteri, ma anche ogni arte magica, profetica, incantatoria.

Lo statuto del daimon è tale da porre un collegamento senza annullare del tutto la distanza: il dio rappresenta la trascendenza che non si mescola (ou meignytai, 203A3) col mondo, l'uomo rappresenta l'immanenza che è il mondo stesso; il daimon, invece, è precisamente rapporto: homilia kai dialektos, relazione e colloquio, che non sono immanenza, né trascendenza, ma, appunto, collegamento, vale a dire congiunzione e distinzione, diairesis e synagogé (Phaedr.266B), ed anche scambio di immagini, vigili e oniriche (Symp. 203A).

Nel celeberrimo racconto della nascita da Poros e Penia è possibile far emergere altri elementi relativi ad una leggibilità linguistica di Eros: concepito nel giorno della nascita di Afrodite, il daimon della comunicazione tra gli uomini e gli dèi discende da Metis, l'intelligenza acuta. La sua nascita in territorio divino è umanamente condizionata dal materializzarsi - sulla scena della festa - di Penia, che porterà nella semantica erotica il tratto dell'indigenza ${ }^{26}$, tratto che eros condivide con logos, mancando entrambi del loro oggetto ${ }^{27}$. L'oggetto del desiderio di eros è l'oggetto del desiderio di logos: la sapienza e la bellezza, ciò che sta lì: intellegibile, perfetto, beato. Esiste una tensione che abita ogni linguaggio e tale tensione è a possedere ciò che esso inseguendo esprime. Si potrebbe - dice Diotima - ricostituire una corretta semantica di tutte le forme di creatività espressiva, di tutte le tensioni al bene e alla felicità, ma gli umani, "separando una certa forma di eros", danno solo ad essa il nome dell'intero" (205B). Gli umani desiderano partorire (tiktein) e il partorire, nel discorso di Diotima, è metafora dell'esprimere. Eros è eros genneseos (206E7), desiderio di generazione, e la generazione cui qui si allude è in primo luogo quella linguistica, i suoi esempi primari sono infatti quelli relativi ad una creazione psichica - ciò che spetta all'anima concepire e partorire (209A3) - quella che nasce dallo studio, dalla poesia, dalla legislazione (208A, 209D), l' esistenza delle quali testimonia come la natura umana non sia del tutto diversa da quella divina. Come il tempo è immagine mobile dell'eterno, secondo la celeberrima espressione del Timeo, l'uomo è immagine mobile del divino e la sua immortalità è un'immortalità umana: la maniera umana di essere divini (208A-B). È toccando il bello (haptomenos tou kalou) e conversando (homilon) con l'amato che ogni uomo gravido nell'anima può partorire figli immortali (209C).

I misteri erotici, quelli che insegnano a desiderare non le cose, ma le forme che abitano le cose, confermano la natura linguistica del percorso segnato dalla scala amoris: strada in salita, che guadagna gradualmente l'essenza di ciò che cerca, passando dal corpo (soma) al comportamento (epithedeuma), all'insegnamento (mathema), alla forma una (monoeides) (211C-E), configurando un passaggio non dal concreto all'astratto, ma dal visibile all'invisibile, dall'apparente all'autentico, dall'esteriore all'interiore, dall'immagine al modello ${ }^{28}$.

L'arrivo di Alcibiade, come è a tutti noto, è l'ultima svolta del dialogo. Ubriaco, Alcibiade dice la verità $(214 \mathrm{E} 6)^{29}$, e ciò che dice (di Eros che per lui è Socrate) è che Socrate vince tutti nei discorsi sempre $(213 \mathrm{E})$, che Socrate è come un linguaggio, come un sogno: da decifrare; possiede un senso nascosto in contrasto con la sua apparenza. Incanta con le nude parole (psilois logois). Le sue nude parole incantano anche se è un altro a pronunciarle, incantano, cioè, in sé, non a causa dell'artificio retorico di chi le pronuncia. È come se non si trattasse di discorsi di Socrate, ma di Socrate stesso diventato 
discorso, linguaggio, parola che rende invasati. I verbi che usa Alcibiade per descriverne gli effetti sono infatti quelli dei katechomenoi, indicano turbamento, sconvolgimento, qualcosa di simile al delirio coribantico (215E). Dai discorsi sgorgano lacrime (215E2-3), essi mettono in subuglio e rendono schiavi gli ascoltatori. Da Socrate, dall'ascolto dei suoi discorsi, si fugge come dalle Sirene (216A7-8). Come da un flauto. Dentro di sé egli possiede divine immagini (agalmata) ${ }^{30}$, immagini tali da costringere all'obbedienza (217A1-3). Immagini da ascoltare. Discorsi che mordono l'anima più di una vipera e costringono a fare e a dire $(218 \mathrm{~A} 7)^{31}$. Non soltanto le cose che dice Alcibiade su Socrate valgono senza distinzione per "lui e i suoi discorsi" (kai autos kai oi logoi autou) (Cfr.221D2-3, D6-7.), ma alla fine del dialogo Alcibiade, operando un'esplicita identificazione tra Socrate e i suoi discorsi - identificazione che sancisce definitivamente la natura linguistica dell' eros 0 , ciò che è lo stesso, la natura erotica del linguaggio - ripete per i sokratikoi logoi lo stesso paragone già presentato per Socrate stesso:

Ma ecco anche questo ho tralasciato di divi all'inizio. Che pure i suoi discorsi sono similissimi alle statuine di sileni che si aprono. Se infatti uno volesse ascoltare $i$ discorsi di Socrate, gli apparirebbero inizialmente del tutto ridicoli, di tali termini ed espressioni sono avwolti da fuori, come la pelle di un satiro tracotante. E infatti parla di asini da soma e di fabbri e di calzolai e di conciapelli, e sembra che dica sempre le stesse cose con le stesse parole, tanto che ogni uomo inesperto e ignorante finirebbe per deridere i suoi discorsi. Ma se uno li vede aperti e entra in essi ${ }^{32}$, innanzitutto scoprirà che sono $i$ soli a possedere all'interno un intelletto, e poi che sono divinissimi e possiedono in sé moltissime immagini di virtù e che tendono verso ciò che è più grande, o meglio verso tutto quel che spetta ricercare a chi voglia diventare bello e buono (221D8-222A6).

\section{REFERÊNCIAS BIBLIOGRÁFICAS}

CALAME, C. (1992) I Greci e l'eros. Simboli pratiche e luoghi. Bari Laterza.

CASERTANO, G. (1997). “Il (in) nome di Eros. Una lettura del discorso di Diotima nel Simposio platonico"; In. "Elenchos" XVIII 2, p. 277-310.

CERRI, G. (2000). “Dalla dialettica all'epos”; In. La struttura del dialogo platonico, Napoli, Loffredo, p. 7-34, p. 25.

DESCHOUX, M. (1980) Platon ou le jeu philosophique. Paris, Les Belles Lettres.

FASCE, S. (1977) Eros. La figura e il culto. Genova, Tilgher.

FOUCAULT, M. (1996) Discorso e verità nella Grecia antica. Roma, Donzelli. (titolo originale Discourse and Truth. The Problematization of Parresia).

CORRIGAN, K. \& CORRIGAN, E. (2004) Plato's Dialectic at Play: Structure, Argument and Myth in Plato's Symposium. Penn State Press.

LACAN, J. (2008) Il seminario. Libro VIII. Il transfert (19601961). Torino, Einaudi 2008 (prima edizione 1991. Edizione originale 1991, Seuil, Paris).

LEDESMA. (2008). “Le sophiste et les exemples; sur le problème de la ressemlance dans le Sophiste de Platon"; In. Revue de Philosophie ancienne XXVI, p. 1-36.

MORAVIA, S. (1995). “Esistenza e passione”; In. Storia delle passioni. Roma-Bari: Laterza, p. 3-38.

MORONCINI, B. (2010) Sull'amore. Jacques Lacan e il Simposio di Platone. Napoli, Cronopio, II edizione.

NUCCI. M. (2009) Platone, Simposio, traduzione e commento, (introduzione di B.Centrone). Torino, EinaudI.

NUSSBAUM, M. (2004) La fragilità del bene. Bologna, Il Mulino. (ed. originale Cambridge, Cambridge University Press 1986).

PALUMBO, L. (2003). “Le emozioni e il pensiero nel Fedone di Platone"; In. La filosofia e le emozioni. Atti del XXXIV Congresso Nazionale della Società Filosofica Italiana. Firenze: Le Monnier.

PIMENTA, M. Platão, pensador da diferença. Uma leitura do Sofista. Belo Horizonte: UFMG, 2006.

PRICE, A. W. (1989) Love and friendship in Plato and Aristotle. Oxford, Clarendon Press.

PUCHNER, M. (2010) The drama of ideas. Platonic provocations in Theater and philosophy. 0xford, 0xford University Press.

RUDHARDT, J. (1999) Eros e Afrodite. Milano, Boringhieri. (titolo originale Le rôle d'Eros et d'Afrodite dans les cosmogonies grecques, Paris, Puf, 1986).

SHEFFIELD, F. (2006) Plato's Symposium: The Ethics of Desire. Oxford, Oxford University Press.

SZLEZÁK, A. (1991) Come leggere Platone. Milano, Rusconi.

Recebido em fevereiro de 2012 Aprovado em maio de 2012. unificati. In questa prospettiva è possibile comprendere come il singolo corpo bello che si viene invitati ad abbandonare non sia un' unità ma solo il frammento di una bellezza unitaria, che va ricostruita. A mano a mano che aumentano gli elementi molteplici unificati dalla denominazione unitaria della bellezza, l'unità del riferimento eidetico, mediato dall'unicità della denominazione, diviene palesemente più chiara.

29. Alcibiade parla con parrhesia (222C2). Sulla parrhesia Foucault (1996), pp. 48-49.

30. Raccoglie intorno a questo termine la sua interpretazione del dialogo Lacan 2008.

31. Come ricorda Nussbaum (2004), p. 332, "un uomo che morì colpito da una freccia definisce le parole d'amore dardi o fulmini che feriscono l'anima".

32. La penetrazione nel discorso è un'altra delle metafore che da sempre consentono di sovrapporre la semantica erotica a quella linguistica, espressiva, ermeneutica. Amare è comprendere, decifrare, penetrare il senso. Amare è penetrare l'intelletto che si nasconde nelle cose. 